\title{
Identification of anti-tuberculosis agents that target the cell-division protein FtsZ
}

\author{
Yuan Lin ${ }^{1}$, Ningyu $\mathrm{Zhu}^{2}$, Yanxing $\mathrm{Han}^{1}$, Jiandong Jiang ${ }^{1}$ and Shuyi Si ${ }^{2}$ \\ Antibiotic resistance to Mycobacterium tuberculosis is a growing problem. Therefore, development of new anti-tuberculosis \\ antibiotics is urgent for the control of tuberculosis (TB) infections. FtsZ, the homolog of eukaryotic tubulin, is a GTPase that \\ assembles into cytokinetic $Z$ rings essential for cell division in prokaryotic cells. FtsZ (filamentous temperature-sensitive \\ protein Z) polymerizes in a GTP-dependent manner, and polymerization of FtsZ forms into dynamic protofilaments. In this study, \\ we screened 20000 compounds to identify inhibitors of GTPase activity of $M$. tuberculosis FtsZ. We found that 297F inhibited \\ GTPase and polymerization of FtsZ, and reduced the amount of FtsZ polymers. Furthermore, 297F has anti-TB activity with low \\ cytotoxicity and shows no antibacterial activities toward other Gram-positive or Gram-negative strains. In vitro, 297F also \\ induced filamentation in Mycobacterium smegmatis. All results suggest that $297 \mathrm{~F}$ inhibits bacterial proliferation by targeting \\ M. tuberculosis FtsZ and it may be useful as a lead compound for developing anti-TB agents.
}

The Journal of Antibiotics (2014) 67, 671-676; doi:10.1038/ja.2014.89; published online 2 July 2014

\section{INTRODUCTION}

Tuberculosis (TB) is a common infectious disease that claims about 2 million lives worldwide. The antibiotics currently available to treat $\mathrm{TB}$ are limited. Recently, HIV epidemics combined with the emergence of multidrug-resistant (MDR-TB) and extensive-resistant (XDR-TB) have aggravated a TB resurgence. ${ }^{1}$ Thus, it is necessary to develop new antibiotics with new molecular targets to effectively treat TB.

FtsZ (filamentous temperature-sensitive protein $\mathrm{Z}$ ) is a protein key to bacterial cell-division protein. It is present in almost all prokaryotes and is the homolog of tubulin in eukaryotes. ${ }^{2}$ However, FtsZ shares only $10 \%$ of its sequence identity at the protein level with tubulin. FtsZ has been shown to possess GTPase activity. It binds and hydrolyzes GTP. ${ }^{3,4}$ After GTP binding and subsequent GTPase activity, FtsZ self-assembles. FtsZ polymerizes in a GTP-dependent manner to form a ' $Z$ ring', which is required for bacterial cell division. GTP hydrolysis and polymerization of FtsZ have been shown to be at the root of the Z-ring dynamics. ${ }^{5,6}$ The recruitment of several other cell-division proteins leads to Z-ring contraction, resulting in septum formation and eventually cell division. In the absence of FtsZ, bacterial cell division is inhibited, although DNA replication and nucleoid segregation occur normally. Such a condition leads to a filamentous phenotype and eventually cell death. Therefore, FtsZ is an especially promising target for new antimicrobial drugs because of its central role in bacterial cell division. ${ }^{7}$

Mycobacterium tuberculosis FtsZ, a $40-\mathrm{kD}$ protein, has been studied both functionally and structurally. Leung et al. ${ }^{8}$ reported the crystal structures of M. tuberculosis FtsZ and its GDP and GTP complexes. The polymers of $M$. tuberculosis FtsZ are more stable and polymerize slower than Escherichia coli FtsZ. ${ }^{9}$ Moreover, the low degree of sequence homology between M. tuberculosis FtsZ and tubulin makes it possible to find compounds specific for M. tuberculosis. Previous studies have shown that inhibition of FtsZ polymerization by small molecules leads to the blockage of bacterial division. ${ }^{10,11}$ However, there are very few studies on inhibitors of $M$. tuberculosis FtsZ. Structurally modified compound SRI-3072, a tubulin polymerization inhibitor, was found to reduce the growth of M. tuberculosis. ${ }^{12}$ Totarol has been shown to inhibit M. tuberculosis, which was determined by its ability to increase the length of Bacilli. ${ }^{13}$

In the present study, the GTPase activity of FtsZ was used to screen 20000 compounds with different structures. We found compound 297F inhibits GTPase activity and FtsZ assembly in vitro, and finally resulted in cell proliferation inhibition in Mycobacterium smegmatis. Interestingly, 297F has anti-TB activity and is bactericidal. The low cytotoxicity to mammalian cells and high MICs on other Grampositive as well as Gram-negative strains made 297F a promising lead anti-TB compound. In addition, our results support the idea that FtsZ may be an excellent target of new anti-TB drugs.

\section{MATERIALS AND METHODS}

Materials

GTP, isopropyl- $\beta$-D-thiogalactopyranoside, malachite green, ammonium molybdate, HEPES, fluorescein diacetate were from Sigma company

\footnotetext{
${ }^{1}$ State Key Laboratory of Bioactive Substances and Function of Natural Medicine, Institute of Materia Medica, Chinese Academy of Medical Sciences and Peking Union Medical College, Beijing, China and ${ }^{2}$ Institute of Medicinal Biotechnology, Chinese Academy of Medical Sciences and Peking Union Medical College, Beijing, China

Correspondence: Professor J Jiang, State Key Laboratory of Bioactive Substances and Function of Natural Medicine, Institute of Materia Medica, Chinese Academy of Medical Sciences and Peking Union Medical College, Beijing 100050, China.

E-mail: jiang.jdong@163.com

or Professor S Si, Institute of Medicinal Biotechnology, Chinese Academy of Medical Sciences and Peking Union Medical College, Tian Tan Xi Li No.1, Beijing, China. E-mail: sisyimb@hotmail.com

Received 20 February 2014; revised 27 May 2014; accepted 28 May 2014; published online 2 July 2014
} 
(Sigma Aldrich, St Louis, MO, USA). Compound 297F was purchased from J\&K Chemical (J\&K Chemical Company, Beijing, China, synthesized by Enamine). The bacterial strains used in the MIC test were clinical isolates or purchased from the American Type Culture Collection (ATCC, Manassas, VA, USA). All other chemicals used were of analytical grade.

\section{FtsZ expression and purification}

M. tuberculosis $\mathrm{H} 37 \mathrm{Rv}$ genomic DNA was provided by the Beijing Research Institute for Tuberculosis Control. The M. tuberculosis fts $Z$ gene was amplified using primers designed from Primer 5.0. The primers used were $5^{\prime}$-TCCATATGACCCCCCCGCACAACTACCTGG-3' (NdeI, sense) and 5'-CG GGATCCTTAGCGGCGCATGAAGGGCGGCACGTC-3' (BamHI, anti-sense).

For the expression of $M$. tuberculosis FtsZ, the PCR product was cloned into the NdeI and BamHI sites of pET16b. The recombinant plasmid pET16b-FtsZ was constructed, with a $6 \times$ His-tag at the N-terminal. The plasmid was transformed into the E. coli BL21 (DE3) strain. A single clone was grown at $37^{\circ} \mathrm{C}$ in LB medium containing $100 \mu \mathrm{g} \mathrm{ml}^{-1}$ ampicillin. The expression of FtsZ was induced by addition of isopropyl- $\beta$-D-thiogalactoside at $30^{\circ} \mathrm{C}$ for $8 \mathrm{~h}$.

The supernatant containing target protein was collected and loaded onto a $\mathrm{Ni}^{2+}$ HisTrap chelating column (GE Health, Milwaukee, WI, USA). The protein was then eluted by a stepwise imidazole gradient in elution buffer ( $25 \mathrm{~mm}$ Tris, $500 \mathrm{~mm} \mathrm{NaCl}, 50-500 \mathrm{~mm}$ imidazole, $\mathrm{pH}$ 7.8). Eluted fractions were analyzed by $12 \%(\mathrm{wt} / \mathrm{vol})$ SDS-polyacrylamide gel electrophoresis (SDSPAGE) followed by Coomassie Blue staining, and the purity was also evaluated. The FtsZ concentration was measured by the Bradford method using bovine serum albumin as a standard. The purified protein was frozen and stored at $-80{ }^{\circ} \mathrm{C}$.

\section{GTPase assay}

The GTPase activity of M. tuberculosis FtsZ was assessed in 96-well plates by determining the ability of the enzyme to liberate inorganic phosphate from GTP. Briefly, M. tuberculosis FtsZ $(10 \mu \mathrm{M})$ was incubated with increasing concentrations of GTP $(0-2000 \mu \mathrm{M})$ in reaction buffer $(50 \mathrm{~mm}$ Tris, $5 \mathrm{~mm}$ $\mathrm{MgCl}_{2}$ ) at $37^{\circ} \mathrm{C}$ for $15 \mathrm{~min}$. The reaction was stopped by the addition of an acidic solution (malachite green, ammonium molybdate and polyvinyl alcohol), and then the activity was detected at $650 \mathrm{~nm}$. Bovine serum albumin was used as a control. A standard curve was run concurrently with each experiment, and GTPase activity is expressed as nmol inorganic phosphate liberated per min per mg of protein.

\section{Compound library screening}

A library of 20000 compounds was used for screening. These compounds included a combination of synthetic (synthesized by Enamine, Ukraine) and natural products (plant, actinomycetes and fungi) from the Institute of Medicinal Biotechnology. FtsZ $(10 \mu \mathrm{M})$ was incubated with the various compounds at $37^{\circ} \mathrm{C}$ for $30 \mathrm{~min}$. The inhibition of GTPase activity of FtsZ was then assessed as described above, though with $1 \mathrm{~mm}$ GTP added. The $\mathrm{IC}_{50}$ was determined based on the ration of the OD units over the concentration of compounds (log plots) that fits to a variable-slope dose-response equation.

\section{Light scattering assay}

The effect of compound 297F on FtsZ assembly was monitored by a $90^{\circ}$ light scattering assay. Briefly, FtsZ $(10 \mu \mathrm{M})$ was mixed with different concentrations of 297F in $25 \mathrm{~mm}$ HEPES buffer, $\mathrm{pH}$ 6.5, $50 \mathrm{~mm} \mathrm{KCl}$ and $5 \mathrm{~mm} \mathrm{MgCl}_{2}$ at $25^{\circ} \mathrm{C}$ for $15 \mathrm{~min}$. After addition of $1 \mathrm{~mm}$ GTP, the reagents were immediately placed in a cuvette at $37^{\circ} \mathrm{C}$. The polymerization reaction was followed by monitoring $90^{\circ}$ light scattering at $600 \mathrm{~nm}$, using a fluorescence spectrophotometer (Jasco, Tokyo, Japan, FP 6500).

\section{Effect of compound 297F on FtsZ polymers}

FtsZ $(100 \mu \mathrm{M})$ was preincubated with different concentrations $(25-100 \mu \mathrm{M})$ of $297 \mathrm{~F}$ or $1 \%$ DMSO in $25 \mathrm{~mm}$ HEPES buffer, $\mathrm{pH} 6.5,50 \mathrm{~mm} \mathrm{KCl}$ and $5 \mathrm{~mm}$ $\mathrm{MgCl}_{2}$ for $30 \mathrm{~min}$. FtsZ was subsequently polymerized with $1 \mathrm{~mm}$ GTP at $25^{\circ} \mathrm{C}$ for $15 \mathrm{~min}$. After polymerization, the mixtures were pelleted at $227000 \mathrm{~g}$ for $30 \mathrm{~min}$. The pellets were then dissolved in SDS loading buffer. The samples were analyzed by Coomassie Brilliant Blue staining of 15\% SDS-PAGE. The FtsZ bands were quantified using Quantity One software (Bio-Rad, Hercules, CA, USA).

\section{Anti-TB activity of compound $297 \mathrm{~F}$}

Anti-TB H37Rv (ATCC 27294) activity of compound 297F was determined using the microplate Alamar Blue assay. The activities against sensitive clinical strain STB-FJ05349 and STB-FJ05060, drug-resistant clinical isolates XDRFJ05195 and XDR-FJ05120 were analyzed using the same method. The firstline anti-TB drugs rifampin and isoniazid were used as references. The final concentrations of each compound ranged from 0.5 to $256 \mu \mathrm{g} \mathrm{ml}^{-1}$. All M. tuberculosis strains were cultured at $37^{\circ} \mathrm{C}$ in Middlebrook $7 \mathrm{H} 9$ broth (Difco, Franklin Lakes, NJ, USA) supplemented with $0.2 \%$ glycerol and $10 \%$ oleic acid-albumin-dextrose-citric acid until the log-phase of growth. The cells were diluted in Middlebrook $7 \mathrm{H} 9$ broth to $10^{6} \mathrm{CFU} \mathrm{ml}^{-1}$ with various concentrations of antibiotics. The MIC was then measured in sterile 96-well microplates, with the final volume of each well at $100 \mu \mathrm{l}$. The visual MIC was defined as the lowest drug concentration that prevented the color change of Alamar Blue reagent from blue to pink.

\section{Sensitivity of other bacterial strains to $297 \mathrm{~F}$}

The MICs of compound 297F against ATCC strains and clinical isolates were determined using the agar dilution method recommend by the Clinical and Laboratory Standards Institute (CLSI). Inoculations were adjusted to yield approximately $10^{4} \mathrm{CFU}$ per spot using a multipoint inoculator (Bolney, Sussex, UK) and were incubated at $35^{\circ} \mathrm{C}$ for $18 \mathrm{~h}$. The MICs were determined as the lowest concentration of the compound that inhibited the growth of bacteria on the plate. Levofloxacin was used as the reference drug.

\section{Cell cytotoxicity evaluation}

To determine the cytotoxicity effect of 297F, cells (MRC-5, Hep G2, HT-29, MG-63 and PC-3) were seeded in 96-well culture plates with culture medium at a density of $5 \times 10^{3}$ cells per well in triplicate. Cells were then subjected to treat with $297 \mathrm{~F}$ at concentrations ranging from 0.11 to $220 \mu \mathrm{M}$. After the cells were grown for an additional $24 \mathrm{~h}$, thiazolyl blue tetrazolium bromide reagent was added. Absorbance was then measured at $490 \mathrm{~nm}$. The values were averaged from triplicate wells and $\mathrm{IC}_{50}$ values were calculated using concentration-response curve fitting.

\section{Mode of action: bacteriostatic vs bactericidal mode}

M. smegmatis $\mathrm{mc}^{2} 155$ is a strain close to $M$. tuberculosis but exhibits fast growing and is non-infectious. This strain was grown to early log-phase in Middlebrook $7 \mathrm{H} 9$ broth at $37^{\circ} \mathrm{C}$. The culture was diluted to $5 \times 10^{5} \mathrm{CFU} \mathrm{ml}^{-1}$ in fresh media containing various concentrations of $297 \mathrm{~F}\left(0.5-32 \mu \mathrm{g} \mathrm{ml}^{-1}\right)$. Bacteria were collected at different time points, serially diluted and plated. After incubation at $37^{\circ} \mathrm{C}$ for $48 \mathrm{~h}$, the number of colonies was counted.

\section{Visualization of mycobacteria morphology}

M. smegmatis $\mathrm{mc}^{2} 155$ was inoculated in $7 \mathrm{H} 9$ broth $\left(5 \times 10^{7} \mathrm{CFU} \mathrm{ml}^{-1}\right)$ containing $20 \mu \mathrm{M} 297 \mathrm{~F}(20 \times \mathrm{MIC})$ and grown for $4 \mathrm{~h}$. Then fluorescein diacetate was then added to the growing $M$. smegmatis to a final concentration of $200 \mu \mathrm{g} \mathrm{ml}^{-1}$. After $10 \mathrm{~min}$, cells were observed using a microscope (Nikon, Tokyo, Japan, TE2000) with a $\times 40$ objective.

\section{RESULTS}

\section{GTPase activity assay of FtsZ}

FtsZ is known to have GTPase activity, thus we measured the activity of the M. tuberculosis FtsZ used in this study. First, we purified the FtsZ. The recombinant protein was expressed mainly in the soluble form when induced by isopropyl- $\beta$-D-thiogalactoside at $30^{\circ} \mathrm{C}$. After purification by Ni-NTA affinity chromatography, only one single band appeared, as determined by Coomassie Blue stained SDS-PAGE. Recombinant FtsZ protein had a molecular mass of approximately $40 \mathrm{kDa}$ (Figure 1a). To determine the GTP-hydrolyzing ability of FtsZ, 

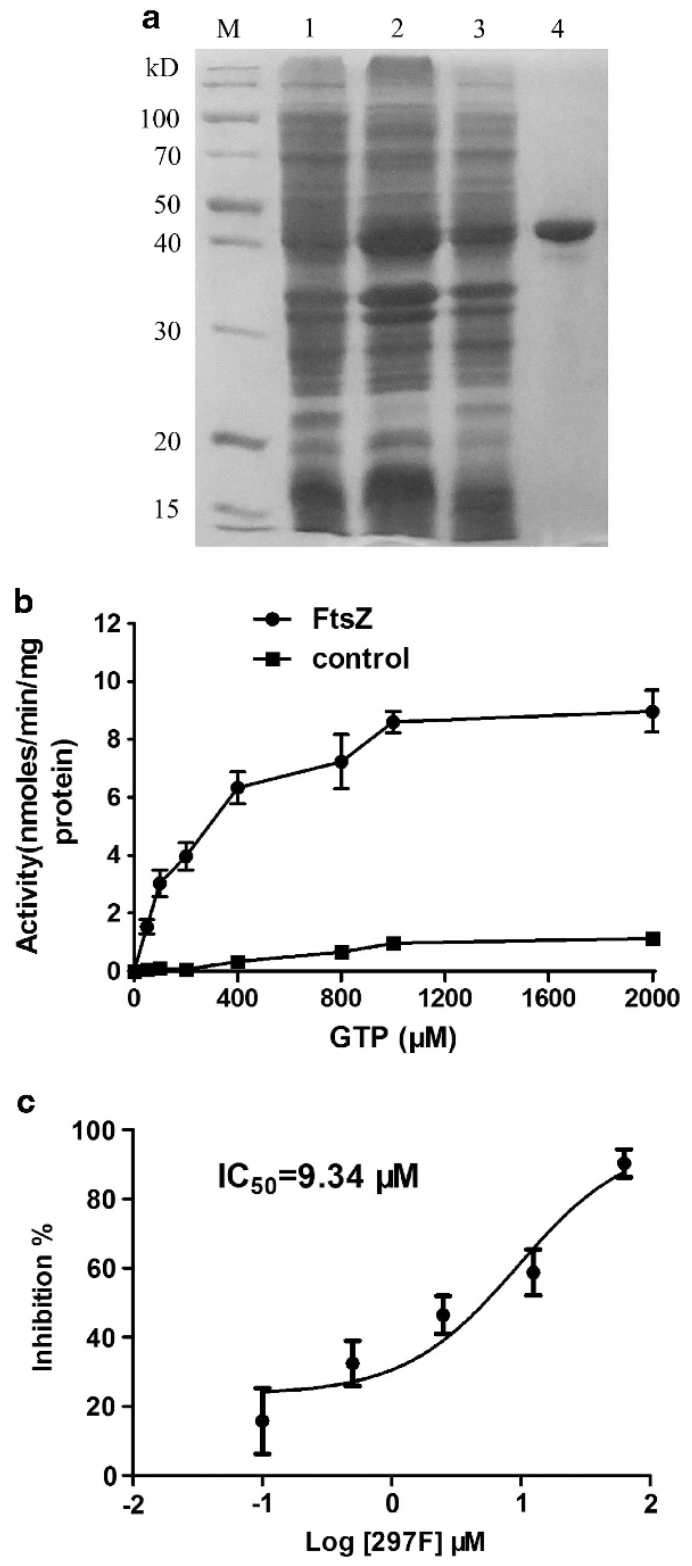

Figure 1 GTPase activity of FtsZ and inhibition of this activity by 297F. (a) Expression and purification of $M$. tuberculosis FtsZ. Protein samples were separated by SDS-polyacrylamide gel electrophoresis and the bands were shown after Coomassie Blue staining. Lane M, protein marker; lane 1, total cell proteins before induction; lane 2, total proteins after induction; lane 3, cellular lysate supernatant after induction; line 4, purified Histagged FtsZ. (b) The GTPase activity of FtsZ was assayed ( $10 \mu \mathrm{m}$ protein per reaction) at $37^{\circ} \mathrm{C}$ with the indicated amounts of substrate. The specific activity of protein in nmol of inorganic phosphate liberated per min per mg is plotted against the substrate concentration. (c) Inhibition of GTPase activity by $297 \mathrm{~F}$. FtsZ $(10 \mu \mathrm{m})$ was incubated with different concentrations of $297 \mathrm{~F}$ for $30 \mathrm{~min}$ and GTPase activity was then determined as mentioned above. The $I_{50}$ value is plotted as the ration of the $O D$ units over the concentration of compounds (log plots) that fits to a variable-slope doseresponse equation. The experiment was repeated three times.

it was incubated with increasing concentrations of GTP $(0-2000 \mu \mathrm{M})$. Upon addition of GTP, FtsZ showed an increase in phosphateliberating activity compared with that of the bovine serum albumin control (Figure 1b).

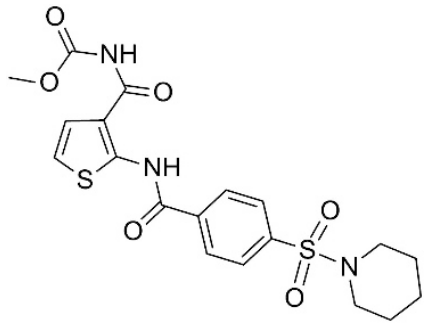

Figure 2 Structure of 297F. (methyl \{2-[4-(piperidin-1-yl-sulfonyl) benzamide] thiophene-3-carbonyl\}carbamate).

Compounds that inhibit the GTPase activity of FtsZ

To screen for compounds that inhibit the GTPase activity of FtsZ, various compounds at $10 \mu \mathrm{g} \mathrm{ml}^{-1}$ were incubated with FtsZ. From the compound library screened, we found 297F inhibited GTPase activity in a dose-dependent manner, with an $\mathrm{IC}_{50}$ value of $9.34 \mu \mathrm{M}$ (Figure 1c). This indicated that the GTPase activity was strongly inhibited by 297F (methyl \{2-[4-(piperidin-1-yl-sulfonyl)benzamide] thiophene-3-carbonyl\} carbamate). Therefore, we chose $297 \mathrm{~F}$ for further investigation. The structure of $297 \mathrm{~F}$ is shown in Figure 2.

\section{Effect of compound 297F on FtsZ polymerization}

As GTP hydrolysis of FtsZ has been shown to be at the root of polymerization, we examined the effects of compound $297 \mathrm{~F}$ on the rate and extent of FtsZ polymerization using $90^{\circ}$ light scattering. As seen in Figure $3 \mathrm{a}$, there was an increase in light scattering upon addition of $1 \mathrm{~mm}$ GTP, which reached a plateau at about $10 \mathrm{~min}$. After $297 \mathrm{~F}$ was added, the light scattering intensity of FtsZ was found to be remarkably decreased. We found that $50 \mu \mathrm{m} 297 \mathrm{~F}$ decreased the light scattering intensity of FtsZ assembly to almost $50 \%$ than that of the control (in the absence of compound 297F), indicating that $297 \mathrm{~F}$ inhibits the assembly and bundling of FtsZ protofilaments. In the presence of $100 \mu \mathrm{M} 297 \mathrm{~F}$, the initial light scattering intensity of assembly was weaker than that of the control, suggesting that $297 \mathrm{~F}$ inhibits the initial rate of FtsZ assembly (Figure 3a).

To directly assess the effects of $297 \mathrm{~F}$ on assembly of FtsZ, we measured the amount of steady-state polymer mass. Specifically, we examined whether 297F could prevent the assembly of FtsZ in the polymerization buffer. The results showed that $50 \mu \mathrm{M} 297 \mathrm{~F}$ significantly reduced the polymers of FtsZ in sediment (Figure $3 \mathrm{~b}$ ). The polymers observed in SDS-PAGE were reduced over 50\% in the presence of $100 \mu \mathrm{M}$ 297F. Therefore, 297F reduced the sedimentation of FtsZ in the reactions, indicating a destabilizing effect of $297 \mathrm{~F}$ on polymer assembly.

\section{Anti-TB activity of compound 297F}

We speculated that the GTPase activity of FtsZ would be essential for cell division and its inhibition would lead to the death of $M$. tuberculosis. Indeed, compound $297 \mathrm{~F}$ showed anti-TB activity with a MIC of $4.4 \mu \mathrm{M}$ for the standard strain H37Rv (Table 1). In wild-type clinical strains (STB-FJ05349 and -FJ05060), the MIC range was 8.8-35.2 $\mu$. For the clinical MDR strains (XDR-FJ05195 and -FJ05120), 297F showed a potency with a range 17.6-35.2 $\mu \mathrm{M}$. This indicated that $297 \mathrm{~F}$ had anti-TB activity comparable to the first-line drugs.

Because we used M. tuberculosis FtsZ for the drug screen, the identified compound may show stronger growth inhibition of this species. Thus, we examined the growth inhibition of 13 bacterial strains from both clinical isolates and the ATCC by 297F. Results 


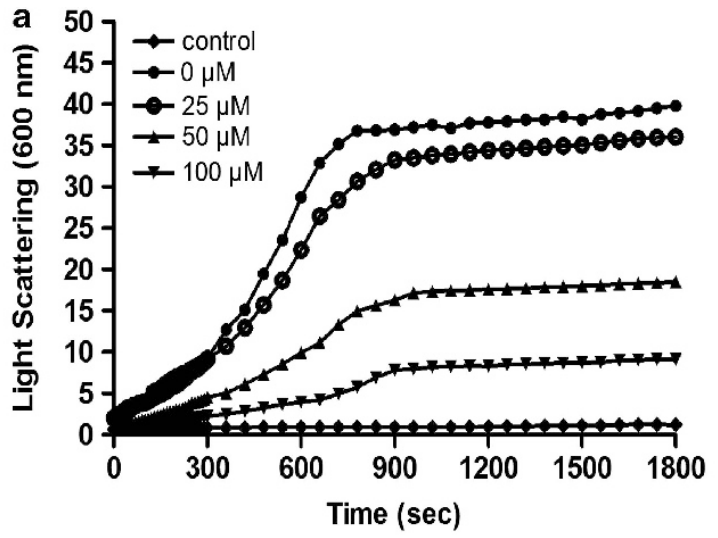

b

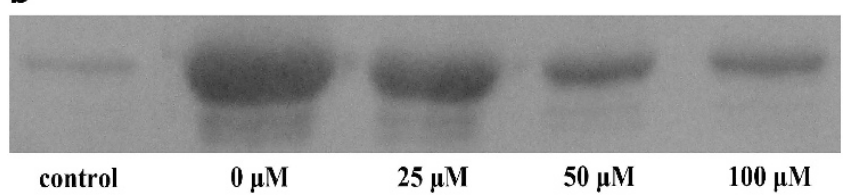

Figure 3 Effect of 297F on FtsZ polymerization. (a) $297 \mathrm{~F}$ inhibited the rate and extent of FtsZ assembly. FtsZ $(10 \mu \mathrm{m})$ was polymerized with $25 \mathrm{~mm}$ HEPES, $50 \mathrm{~mm} \mathrm{KCl}, 5 \mathrm{~mm} \mathrm{MgCl} 2$ and $1 \mathrm{~mm}$ GTP in the absence or presence of different concentrations $(0-100 \mu \mathrm{m})$ of 297F. The control indicates FtsZ polymerizes without GTP and compound. The rate and extent of the assembly reaction were monitored at $600 \mathrm{~nm}$. (b) $297 \mathrm{~F}$ prevented assembly of FtsZ polymers. FtsZ $(100 \mu \mathrm{m})$ was preincubated with different concentrations $(0-100 \mu \mathrm{m})$ of $297 \mathrm{~F}$. FtsZ polymerizes without compound and GTP was shown as the control. After addition of $1 \mathrm{~mm}$ GTP for $15 \mathrm{~min}$, the polymers were pelleted at $227000 \mathrm{~g}$ for $30 \mathrm{~min}$. The pellets were then analyzed by $15 \%$ SDS-polyacrylamide gel electrophoresis and the FtsZ bands were visualized by Coomassie Brilliant Blue staining.

showed that 297F had high MICs on both Gram-positive and Gramnegative strains (MIC $>70 \mu \mathrm{M}$; Table 1 ).

Moreover, the $\mathrm{IC}_{50}$ of cytotoxicity for normal lung fibroblast MRC- 5 cells was found to be $55 \mu$ m. For cancer cells Hep G2, HT29 , MG-63 and PC-3, the $\mathrm{IC}_{50}$ were all $\geqslant 55 \mu \mathrm{m}$. These indicated that the compound 297F exhibited promising anti-TB activity without appreciable cytotoxicity.

Mechanism of M. tuberculosis growth inhibition by 297F

Compound 297F may inhibit the growth of TB through bacteriostatic or bactericidal effects. As M. tuberculosis grows slowly, we chose M. smegmatis, a nonpathogenic strain biologically close to M. tuberculosis, to examine viability after treatment with 297F. The MIC of 297F for M. smegmatis was $1.1 \mu \mathrm{m}$. Interestingly, 297F showed bactericidal activity against this strain at the MIC and the activity was increased dramatically when the concentrations were at $4 \times, 16 \times$ and $64 \times$ MIC (Figure 4). The results suggest a strong bactericidal activity of $297 \mathrm{~F}$ against mycobacteria.

\section{F inhibited cell proliferation}

As $297 \mathrm{~F}$ inhibits the assembly and bundling of FtsZ protofilaments, we speculate that it may induce filamentation in mycobacteria. Fluorescein diacetate is a cell dye that can enter the integral cell membrane. ${ }^{14}$ Thus, we examined the effect of $297 \mathrm{~F}$ on the morphology of $M$. smegmatis by comparing the fluorescence of fluorescein diacetate on the strain in the absence and presence of
Table 1 MICs of $297 F$ against various strains

\begin{tabular}{|c|c|c|c|c|}
\hline \multirow[b]{2}{*}{ Strain } & \multicolumn{4}{|c|}{$M I C(\mu M)$} \\
\hline & $297 F$ & Rifampin & Isoniazid & Levofloxacin \\
\hline H37Rv & 4.4 & 0.3 & 1.8 & - \\
\hline STB-FJ05349 & 8.8 & 0.6 & 3.6 & - \\
\hline STB-FJ05060 & 35.2 & 0.6 & 3.6 & - \\
\hline XDR-FJ05195 & 35.2 & $>311$ & 29.2 & - \\
\hline XDR-FJ05120 & 17.6 & $>311$ & 29.2 & - \\
\hline Staphylococcus aureus $29213^{a}$ & $>70$ & - & - & 0.67 \\
\hline Staphylococcus aureus $33591^{\mathrm{b}}$ & $>70$ & - & - & 0.67 \\
\hline Staphylococcus epidermidis $12228^{c}$ & $>70$ & - & - & 21.6 \\
\hline Staphylococcus epidermidis $12-10^{d}$ & $>70$ & - & - & 10.8 \\
\hline Enterococcus faecalis $29212^{\mathrm{e}}$ & $>70$ & - & - & 2.7 \\
\hline Enterococcus faecalis $51299^{f}$ & $>70$ & - & - & 1.3 \\
\hline Enterococcus faecium $700221^{\dagger}$ & $>70$ & - & - & 172.8 \\
\hline Enterococcus faecium $09-10^{e}$ & $>70$ & - & - & 172.8 \\
\hline Escherichia coli $25922^{\mathrm{g}}$ & $>70$ & - & - & $\leqslant 0.1$ \\
\hline Escherichia coli 09-1 ${ }^{\mathrm{h}}$ & $>70$ & - & - & 10.8 \\
\hline Klebsiella pneumonia $700603^{\mathrm{h}}$ & $>70$ & - & - & 5.4 \\
\hline Klebsiella pneumonia 09-8g & $>70$ & - & - & 10.8 \\
\hline Pseudomonas aeruginosa 27853 & $>70$ & - & - & 10.8 \\
\hline
\end{tabular}

STB, clinically sensitive strain of $M$. tuberculosis: XDR, extensive drug resistance of M. tuberculosis. Strain FJ05349, FJ05060, FJ05195 and FJ05120 were all clinical isolates of $M$. tuberculosis.

aMSSA, methicillin-sensitive Staphylococcus aureus.

DMRSA, methicillin-resistant Staphylococcus aureus.

"MSSE, methicillin-sensitive Straphylococcus epidermidis.

dMRSE, methicillin-resistant Straphylococcus epidermidis.

eVSE, vancomycin-sensitive enterococcus.

$\mathrm{V} V R E$, vancomycin-resistant enterococcus.

gESBLs $(-)$, extended spectrum $\beta$-lactamases $(-)$.

${ }^{h} \operatorname{ESBLs}(+)$, extended spectrum $\beta$-lactamases $(+)$.

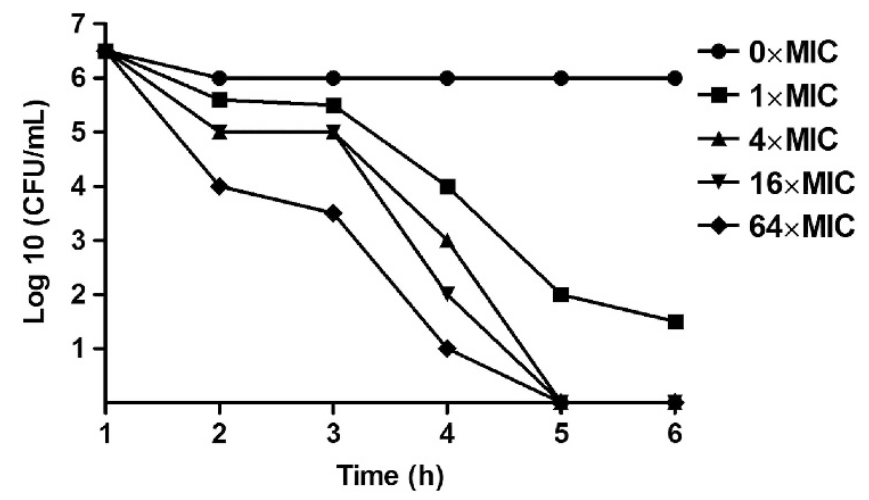

Figure 4 Dose-dependent bactericidal activity of 297F against M. smegmatis $\mathrm{mc}^{2} 155$. The MIC of $297 \mathrm{~F}$ against this strain is $1.1 \mu \mathrm{m}$. Viability of $M$. smegmatis $\mathrm{mc}^{2} 155$ was determined in the presence of $297 \mathrm{~F}$ at 1- to 64-fold of MIC.

297F. Treatment of $297 \mathrm{~F}$ increased the length of M. smegmatis, with elongation of two- to threefold (3-4 to $7-8 \mu \mathrm{m}$ ) (Figure 5). Normally, the assembly of Fts $Z$ forms the backbone of the $\mathrm{Z}$ ring. 297F may inhibit the polymerization of FtsZ into the $Z$ ring, resulting in an imperfection in septum formation (cytokinesis). In addition to the segregated nucleoids, the M. smegmatis showed a remarkable elongation. Therefore, the proliferation of $M$. smegmatis was inhibited by $297 \mathrm{~F}$. 

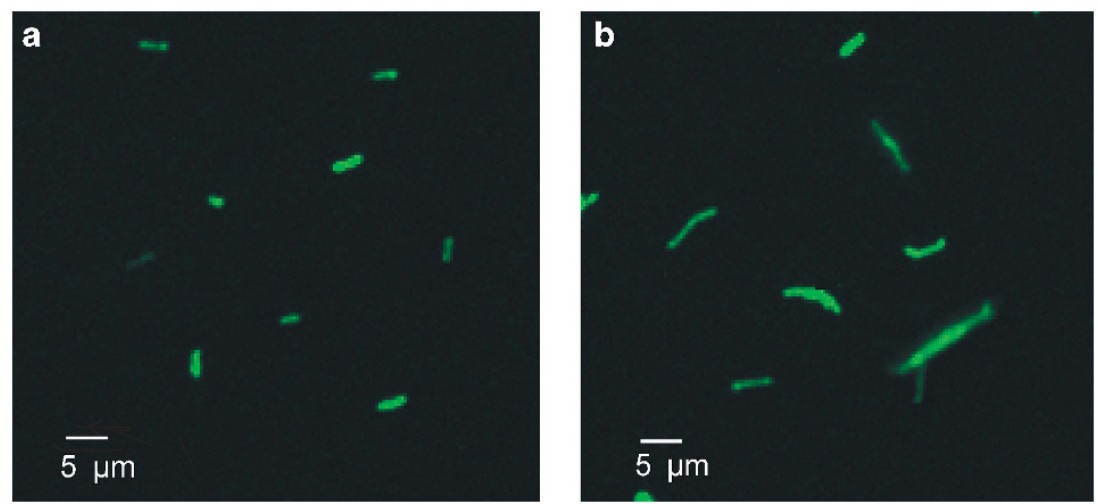

Figure $5297 \mathrm{~F}$ induced filamentation in $M$. smegmatis $\mathrm{mc}^{2} 155$. Cell morphology was observed under a fluorescence microscope. M. smegmatis mc ${ }^{2} 155$ was grown $4 \mathrm{~h}$ in the absence (a) and presence of $20 \mu \mathrm{m} 297 \mathrm{~F}$ (b). The scale bar is $5 \mu \mathrm{m}$.

\section{DISCUSSION}

Development of new anti-TB agents is of critical importance worldwide. Cell division is considered to be one of the important therapeutic targets for antibacterial drugs. ${ }^{15} \mathrm{FtsZ}$ is a protein crucial to bacterial cell division. ${ }^{16}$ In the present study, we focused on FtsZ from M. tuberculosis. By analyzing a compound library, we first found that compound 297F inhibited GTPase activity of FtsZ. Additionally, light scattering and SDS-PAGE results from this study suggest that 297F decreased the FtsZ polymers. More importantly, 297F showed anti-TB activity and had high MICs on other bacterium. Furthermore, 297F inhibited bacterial proliferation in vitro. Collectively, our results suggest that we have identified an anti-TB compound that targets cytoplasmic protein FtsZ.

There are many reports on broad-spectrum inhibitors of FtsZ. ${ }^{7,17,18}$ Because FtsZ is absent from the mitochondria of higher eukaryotes, it has evolutionary distance from tubulin, and a known biochemical activity and atomic structure, FtsZ is an attractive target for developed agents that may cause selective bactericidal effect to bacterial pathogens. ${ }^{12,19}$ However, there are few selective inhibitors of $M$. tuberculosis known to date. It was previously found that totarol increased the length of B. subtilis, suggesting it has inhibitory activity against mycobacterial. Consequently, researchers confirmed that totarol indeed inhibited the proliferation of $M$. tuberculosis. ${ }^{13,20}$ Both SRI-3072 and SRI-7614 are 2-alkoxycarbonylaminopyridines, belonging to a library of tubulin inhibitors. Compounds in this library were previously applied to a $M$. tuberculosis strain to test for anti-TB activity, and ultimately SRI-3072 and SRI-7614 were found to have activity. ${ }^{12}$ To our knowledge, there are no anti-TB agents by screening inhibitors of GTPase activity of M. tuberculosis FtsZ until now. The advantage of the screening method used in our study is more likely to find selective inhibitors.

To our surprise, $297 \mathrm{~F}$ was found to selectively inhibit the growth of M. tuberculosis. As is known, FtsZ is ubiquitous in prokaryotes and archaea, and these organisms all have basically the same structures. Previous studies have shown that FtsZ has two domains, one of which is a GTPase domain and the other is a water-polarizing residue, and the two domains are arranged around a central helix. ${ }^{2,8,21}$ Sequence alignment of the FtsZ structure revealed that most residues involved in GDP binding are conserved, which is different from that found in GTPase. GTP binding of FtsZ involves four phosphate-binding loops and a sugar-binding loop in the first domain, with guanine being recognized by residues in the central connecting helix. Although the structures of FtsZ in bacteria are similar, there are some significant differences between those of E. coli and M. tuberculosis. The
M. tuberculosis FtsZ polymerizes and depolymerizes much more slowly than E. coli, and the formed polymers are more stable. ${ }^{22}$ Further, divalent calcium strongly enhances the assembly of E. coli FtsZ, but has no effect on M. tuberculosis FtsZ. ${ }^{9}$ These findings indicate that the assembly dynamics of $M$. tuberculosis FtsZ are regulated by different mechanisms than other bacteria. We infer that the highly selective activity of $297 \mathrm{~F}$ against $M$. tuberculosis might originate from this diversity.

Among the known anti-TB agents, totarol inhibited the polymerized mass of $M$. tuberculosis by $27 \%$ at $50 \mu \mathrm{M}$ with a MIC of $2 \mu \mathrm{M} .{ }^{13,20}$ SRI-3072 and SRI-7614 inhibited M. tuberculosis GTPase activity by $20-25 \%$ at $100 \mu \mathrm{M}$ with a MIC of $0.15-6.25 \mu \mathrm{g} \mathrm{ml}^{-1} .{ }^{12}$ In this study, 297F inhibited the GTPase activity of FtsZ with an $\mathrm{IC}_{50}$ value of $9.34 \mu \mathrm{M}$ and decreased the FtsZ assembly to almost $50 \%$ at $50 \mu \mathrm{M}$. Thus, the anti-TB activity and selectivity of $297 \mathrm{~F}$ is comparable to that of the known agents. In addition, there was no biological activity report on $297 \mathrm{~F}$ in the previous studies.

The GTPase activity of $M$. tuberculosis FtsZ was inhibited by $50 \%$ in the presence of $9.34 \mu \mathrm{M} 297 \mathrm{~F}$, whereas the growth of M. tuberculosis $\mathrm{H} 37 \mathrm{Rv}$ was inhibited with a MIC of $4.4 \mu \mathrm{M}$. It seems that $297 \mathrm{~F}$ required to inhibit $M$. tuberculosis proliferation was lower than the concentration required to inhibit either GTPase activity or assembly of FtsZ in vitro. A similar difference was observed in several reports. ${ }^{13,23,24}$ The possible reason for this might be that the binding of $297 \mathrm{~F}$ with FtsZ interferes with the binding of FtsZ with its interacting proteins, such as ZipA, FtsA, FtsW. ${ }^{25-27}$ All these interactions could also inhibit the formation and functioning of the cytokinetic $\mathrm{Z}$ ring in bacteria. Another possible explanation is that the intracellular concentration of $297 \mathrm{~F}$ is likely to be much higher than the concentration of the compound in vivo.

We found 297F inhibits the assembly and bundling of FtsZ protofilaments, and increases the cell length of M. smegmatis, suggesting it has anti-proliferative activity. In the bacteria, cell division is engineered by the dynamic of $\mathrm{Z}$ ring. ${ }^{28}$ Recent studies indicated that compounds targeting FtsZ assembly may perturb the $\mathrm{Z}$ ring to inhibit bacterial cytokinesis. ${ }^{13,29}$ We speculate that $297 \mathrm{~F}$ also perturbes the Z-ring formation. However, further investigation is needed to demonstrate this. DNA condensation and nucleoid segregation are also known to be key biological processes in cell division. Several reports have found that inhibitors of FtsZ GTP activity did not perturb DNA condensation and nucleoid segregation. ${ }^{13,24,29}$ In future studies, we are going to determine whether $297 \mathrm{~F}$ affects DNA condensation and nucleoid segregation, contributing to its division inhibition specificity of the $\mathrm{Z}$ ring. 
Tubulin, the eukaryotic homolog of FtsZ, has been successfully used as a target for developing drugs against several diseases, including cancer and fungal diseases. ${ }^{30,31}$ The fact that FtsZ is an essential protein in almost all the prokaryotes makes it an attractive target for developing novel antimicrobial agents. In this study, we found 297F inhibited GTPase activity and assembly of FtsZ and it was selective to $M$. tuberculosis and displayed low toxicity to mammalian cells. Therefore, it is possible that $297 \mathrm{~F}$ may serve as a lead for antibiotic development through chemical modification to optimize inhibitor potency and specificity.

\section{ACKNOWLEDGEMENTS}

This work was supported by the National Natural Science Foundation of China (81302816, 81321004), the Basic Scientific Research Program of Materia Medica, CAMS (2013ZD05), and grants from State Mega Programs (2012ZX09301002-003/006).

1 Dye, C. et al. WHO and the future of disease control programmes. Lancet $\mathbf{3 8 1}$ 413-418 (2013).

2 Lowe, J. \& Amos, L. A. Crystal structure of the bacterial cell-division protein FtsZ. Nature 391, 203-206 (1998).

3 Scheffers, D. J. \& Driessen, A. J. Immediate GTP hydrolysis upon FtsZ polymerization. Mol. Microbiol. 43, 1517-1521 (2002).

4 Oliva, M. A. et al. Assembly of archaeal cell division protein FtsZ and a GTPase-inactive mutant into double-stranded filaments. J. Biol. Chem. 278, 33562-33570 (2003).

5 Romberg, L. \& Levin, P. A. Assembly dynamics of the bacterial cell division protein FtsZ: poised at the edge of stability. Annu. Rev. Microbiol. 57, 125-154 (2003).

6 Bernhardt, T. G. \& de Boer, P. A. The Escherichia coli amidase AmiC is a periplasmic septal ring component exported via the twin-arginine transport pathway. Mol. Microbiol. 48, 1171-1182 (2003).

7 Dasgupta, D. Novel compound with potential of an antibacterial drug targets FtsZ protein. Biochem. J. 423, e1-e3 (2009).

8 Leung, A. K. et al. Structure of Mycobacterium tuberculosis FtsZ reveals unexpected, G protein-like conformational switches. J. Mol. Biol. 342, 953-970 (2004).

9 Jaiswal, R. \& Panda, D. Differential assembly properties of Escherichia coli FtsZ and Mycobacterium tuberculosis FtsZ: an analysis using divalent calcium. J. Biochem. 146, 733-742 (2009).

10 Vollmer, W. The prokaryotic cytoskeleton: a putative target for inhibitors and antibiotics? Appl. Microbiol. Biotechnol. 73, 37-47 (2006)

11 Rai, D., Singh, J. K., Roy, N. \& Panda, D. Curcumin inhibits FtsZ assembly: an attractive mechanism for its antibacterial activity. Biochem. J. 410, 147-155 (2008).
12 White, E. L., Suling, W. J., Ross, L. J., Seitz, L. E. \& Reynolds, R. C. 2-Alkoxycarbonylaminopyridines: inhibitors of Mycobacterium tuberculosis FtsZ. J. Antimicrob. Chemother. 50, 111-114 (2002).

13 Jaiswal, R., Beuria, T. K., Mohan, R., Mahajan, S. K. \& Panda, D. Totarol inhibits bacterial cytokinesis by perturbing the assembly dynamics of FtsZ. Biochemistry 46 , 4211-4220 (2007).

14 Boyd, V., Cholewa, O. M. \& Papas, K. K. Limitations in the use of fluorescein diacetate propidium iodide (FDA/PI) and cell permeable nucleic acid stains for viability measurements of isolated islets of langerhans. Curr. Trends Biotechnol. Pharm 2, 66-84 (2008).

15 Projan, S. J. New (and not so new) antibacterial targets-from where and when will the novel drugs come? Curr. Opin. Pharmacol. 2, 513-522 (2002).

16 Sun, Q. \& Margolin, W. FtsZ dynamics during the division cycle of live Escherichia coli cells. J. Bacteriol. 180, 2050-2056 (1998).

17 Kapoor, S. \& Panda, D. Targeting FtsZ for antibacterial therapy: a promising avenue. Expert. Opin. Ther. Targets 13, 1037-1051 (2009).

18 Kumar, K. et al. Discovery of anti-TB agents that target the cell-division protein FtsZ. Future Med. Chem. 2, 1305-1323 (2010).

19 Wang, J. et al. Discovery of a small molecule that inhibits cell division by blocking FtsZ, a novel therapeutic target of antibiotics. J. Biol. Chem. 278, 44424-44428 (2003).

20 Constantine, G. H., Karchesy, J. J., Franzblau, S. G. \& LaFleur, L. E. (+)-Totarol from Chamaecyparis nootkatensis and activity against Mycobacterium tuberculosis. Fitoterapia 72, 572-574 (2001)

21 Cordell, S. C., Robinson, E. J. \& Lowe, J. Crystal structure of the SOS cell division inhibitor SulA and in complex with FtsZ. Proc. Natl Acad. Sci. USA 100, 7889-7894 (2003)

22 White, E. L. et al. Slow polymerization of Mycobacterium tuberculosis FtsZ. J. Bacteriol. 182, 4028-4034 (2000).

23 Urgaonkar, S. et al. Synthesis of antimicrobial natural products targeting FtsZ: (+ / -) dichamanetin and (+/ -)-2'-hydroxy-5'-benzylisouvarinol-B. Org. Lett. 7, 5609-5612 (2005).

24 Margalit, D. N. et al. Targeting cell division: small-molecule inhibitors of FtsZ GTPase perturb cytokinetic ring assembly and induce bacterial lethality. Proc. Natl Acad. Sci. USA 101, 11821-11826 (2004)

25 Geissler, B., Elraheb, D. \& Margolin, W. A gain-of-function mutation in ftsA bypasses the requirement for the essential cell division gene zipA in Escherichia coli. Proc. Nat Acad. Sci. USA 100, 4197-4202 (2003).

26 Szwedziak, P., Wang, Q., Freund, S. M. \& Lowe, J. FtsA forms actin-like protofilaments. EMBO J. 31, 2249-2260 (2012).

27 Pastoret, S. et al. Functional analysis of the cell division protein FtsW of Escherichia coli. J. Bacteriol. 186, 8370-8379 (2004).

28 Stricker, J., Maddox, P., Salmon, E. D. \& Erickson, H. P. Rapid assembly dynamics of the Escherichia coli FtsZ-ring demonstrated by fluorescence recovery after photobleaching. Proc. Natl Acad. Sci. USA 99, 3171-3175 (2002).

29 Beuria, T. K. Santra, M. K. \& Panda, D. Sanguinarine blocks cytokinesis in bacteria by inhibiting FtsZ assembly and bundling. Biochemistry 44, 16584-16593 (2005)

30 Kavallaris, M. Microtubules and resistance to tubulin-binding agents. Nat. Rev. Cance 10, 194-204 (2010).

31 Okusaga, 0. et al. Association of seropositivity for influenza and coronaviruses with history of mood disorders and suicide attempts. J. Affect. Disord. 130, 220-225 (2011). 